\title{
Limits of Disturbance Rejection for Indirect Control
}

\author{
Vinay Kariwala and Sigurd Skogestad \\ Department of Chemical Engineering, \\ Norwegian University of Science and Technology, N-7491 Trondheim, Norway \\ \{kariwala, skoge\}@chemeng.ntnu.no
}

\begin{abstract}
In many practical problems, the primary controlled variable is not available for feedback and is needed to be controlled indirectly using secondary measurements. We derive bounds on the $\mathcal{H}_{2}$ and $\mathcal{H}_{\infty}$ optimal achievable performance for systems under indirect control, which have all scalar signals. These bounds are useful for gaining insights into the factors posing limitations on the achievable performance. As compared to direct control, the unstable poles can severely limit the control quality for indirect control. The limiting effect of the unstable poles can be reduced using an additional feedforward controller, when the disturbance is measurable.
\end{abstract}

\section{INTRODUCTION}

During the past two decades, a number of algorithms have been proposed for optimal controller design. Before the controller is designed, it is beneficial for an engineer to ascertain whether the process is controllable or in other words, whether it is possible to satisfy the desired performance objectives. Clearly, it is impossible to meet arbitrary performance objectives, as Skogestad and Postlethwaite [1] have remarked, "even the best control system cannot make a Ferrari out of a Volkswagen."

The controller design always involves trade-offs between various competing objectives. Then, the controllability analysis is useful for finding where the effect of the limiting factors can be accommodated easily through appropriate modification of performance requirements. In some cases, the controllability analysis can also suggest process design changes leading to easier operation of the plant; see e.g. $\mathrm{pH}$-control process in [1], [2]. Through some real-life application examples, Stein [3] highlighted the importance of understanding what a controller can or cannot do over purely numerical control design algorithms.

A key step in controllability analysis is to assess whether there exists a stabilizing controller that makes the various sensitivity functions along the closed-loop system sufficiently small. When the existence of such a controller is established, it is ensured that the effect of the disturbances on the various signals can be minimized. For this purpose, Chen [4] provides the achievable values for the weighted sensitivity and complementary sensitivity functions. Kariwala et al. [5] obtained the minimal achievable value of the closed-loop transfer matrix from the disturbances to the control signals or inputs. For an overview of the available results on controllability analysis and fundamental performance limitations, the reader is referred to [1], [6] and [7].

Though useful, the applicability of the available bounds on various closed-loop sensitivity functions is limited to the cases, where the performance is measured in terms of the sensed outputs or control signals. In many practical problems of interest, the primary controlled variables are not measured or are not suitable for feedback due to various reasons, e.g. infrequent availability. For example, in process industries, it is required that the effect of disturbances on the composition variables be minimized. These composition variables are often not measured on-line and are controlled indirectly using secondary measurements, e.g. tray temperatures for distillation columns. Freudenberg et al. [8] provide some other practical examples, where indirect control is necessary.

In this paper, we consider the fundamentals performance limitations of systems under indirect control. Note that disturbance rejection using indirect control is the same as the generalized control problem. The fundamental limitations of feedback control in the generalized control framework has been studied earlier by Freudenberg et al. [8], who obtained many useful results including the Bode and Poisson type integral relationships. Here, we focus on finding the achievable bounds on the closed-loop transfer function from the disturbances to the primary controlled variables ( $d$ and $z$, respectively in Figure 1) in the $\mathcal{H}_{2}$ and $\mathcal{H}_{\infty}$ control frameworks. These bounds characterize the effects of unstable poles and zeros on the quality of disturbance rejection and complement the results in [8]. We also show that as compared to direct control, the unstable poles can severely limit the achievable performance for indirect control. The limiting effect of the unstable poles can be reduced by using a feedforward controller in additional to the feedback controller (two degrees of freedom), whenever the disturbance is measurable. As an offset, the proposed bounds unify the available bounds on various closed-loop sensitivity functions. We only consider cases where all signals in the system are scalar, however, the results can be easily extended to multivariable systems through additional consideration of directions of unstable poles and zeros.

\section{Problem Formulation}

For a given matrix $A \in \mathcal{C}^{m \times n}, A^{H}$ denotes the complex conjugate transpose, $\bar{\sigma}(A)$ denotes the maximum singular value and for $A \in \mathcal{C}^{m \times m}, \rho(A)$ is the spectral radius. We call a square matrix $A$ a positive definite matrix (denoted as $A \succ$ $0)$ if all the eigenvalues of its hermitian part $\left(A+A^{H}\right)$ are positive. Positive semi-definite matrices are defined similarly and are denoted as $A \succeq 0$. For a transfer function $G(s), z$ is called a zero of $G(s)$, if $G(z)=0$. Similarly, $p$ is called a 


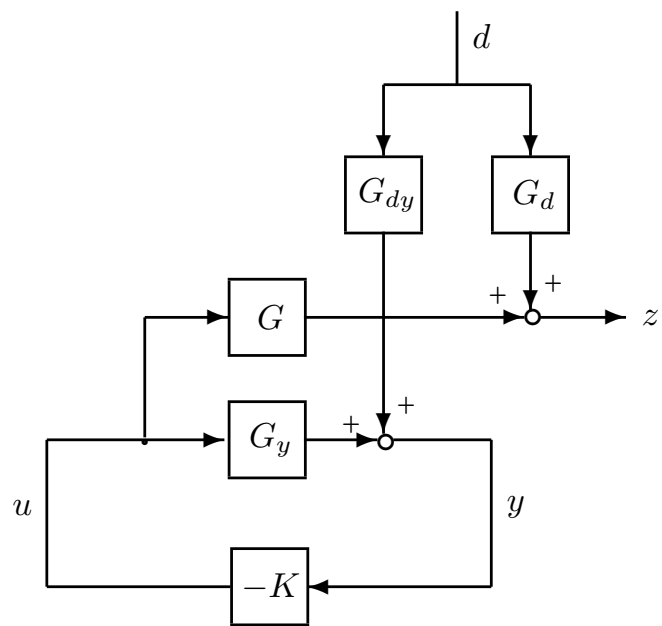

Fig. 1. Closed-loop system under indirect control

pole of $G(s)$, if $G^{-1}(p)=0 . G(s)$ is considered stable, if it does not have any pole in the right half of the complex plane including imaginary axis. The $\mathcal{H}_{\infty}$ norm of stable $G(s)$ is

$$
\|G(s)\|_{\infty}=\sup _{\operatorname{Re}(s)>0}|G(s)|=\sup _{\omega \in \mathcal{R}}|G(j \omega)|
$$

The $\mathcal{H}_{2}$ norm of stable and strictly proper $G(s)$ is

$$
\|G(s)\|_{2}^{2}=\frac{1}{2 \pi} \int_{-\infty}^{\infty}|G(j \omega)|^{2} d \omega
$$

Let $(A, B, C, 0)$ be the state space realization of $G(s)$. Then, $\|G(s)\|_{2}^{2}=C X C^{H}$, where $X$ is the controllability gramian that solves the following Lyapunov equation (see, for example [1])

$$
A^{H} X+X A+B B^{H}=0
$$

In the following discussion, we drop the argument $s$ for notational simplicity, i.e. $G(s)$ is represented as $G . G^{(i)}$ denotes the $i^{\text {th }}$ derivative of $G$ with respect to the complex variable s, i.e. $G^{(i)}=\frac{d^{i} G}{d s^{i}}$ and $G^{(0)}=G$.

With these preliminaries, consider the closed-loop system shown in Figure 1, where $z$ is the primary controlled variable, and $y$ is the secondary variable (measurement) available for indirect control. The model is

$$
\begin{aligned}
z & =G u+G_{d} d \\
y & =G_{y} u+G_{d y} d
\end{aligned}
$$

and $u=-K y$. Define the sensitivity function $S_{y}=(I+$ $\left.G_{y} K\right)^{-1}$. Then,

$$
z=\underbrace{\left(G_{d}-G K S_{y} G_{d y}\right)}_{T_{z d}} d
$$

Define the transfer function from $\left[\begin{array}{ll}d & u\end{array}\right]^{T}$ to $\left[\begin{array}{ll}z & y\end{array}\right]^{T}$

$$
\tilde{G}=\left[\begin{array}{cc}
G_{d} & G \\
G_{d y} & G_{y}
\end{array}\right]
$$

Then, $T_{z d}$ in (1) can also be obtained as a lower linear fractional transformation of $\tilde{G}$ in (1) with $K$.
In the remaining discussion in this paper, we aim at gaining insight into the limitations on the minimal achievable value of $\left\|T_{z d}\right\|_{i}, i=2, \infty$ due to unstable poles and zeros of the system. In general, one may instead want to characterize the achievable value of $\left\|W_{p} T_{z d}\right\|_{i}$, where $W_{p}$ is a frequency dependent weight. The results presented here can easily be extended to handle this case through appropriate choices of $G_{d}$ and $G$.

Assumption 1: We make the following assumptions throughout this paper:

(a) The system is stabilizable, which requires that all unstable poles of $\tilde{G}$ are also poles of $G_{y}$.

(b) All signals in the system are scalar.

(c) $G_{d}$ is stable $\overline{\overline{\sqrt{2}}}$

We note that in some cases, the assumption of stable $G_{d}$ can be limiting, for example, when the disturbances enter through the input channels such that $G_{d}=G$, but it simplifies the analysis considerably. To analyze the effect of unstable or right half plane (RHP) poles and zeros on the achievable performance, we need the following notation:

$\alpha_{i}$ - RHP zeros of $G$, i.e. $G\left(\alpha_{i}\right)=0, i=1, \cdots, n_{\alpha}$.

$\beta_{i}$ - RHP zeros of $G_{d y}$, i.e. $G_{d y}\left(\beta_{i}\right)=0, i=1, \cdots, n_{\beta}$.

$\gamma_{i}$ - RHP poles of $G_{y}$ that are also RHP poles of $G$, i.e. $G_{y}^{-1}\left(\gamma_{i}\right)=G_{2}^{-1}\left(\gamma_{i}\right)=0 ; G_{d y}^{-1}\left(\gamma_{i}\right) \neq 0, i=$ $1, \cdots, n_{\gamma}$.

$\delta_{i}$ - RHP poles of $G_{y}$ that are also RHP poles of $G_{d y}$, i.e. $G_{y}^{-1}\left(\delta_{i}\right)=G_{d y}^{-1}\left(\delta_{i}\right)=0 ; G^{-1}\left(\delta_{i}\right) \neq 0, i=1, \cdots, n_{\delta}$.

$\epsilon_{i}$ - Additional RHP poles of $G_{y}$, i.e. $G_{y}^{-1}\left(\epsilon_{i}\right)=0$; $G^{-1}\left(\epsilon_{i}\right) \neq 0$ and $G_{d y}^{-1}\left(\epsilon_{i}\right) \neq 0, i=1, \cdots, n_{\epsilon}$.

In general, $G_{y}$ can have RHP poles that are also RHP poles of both $G$ and $G_{d y}$. For the system to be stabilizable $G_{y}$ must have these poles with multiplicity of at least 2 . Then, this case can be treated by appropriately partitioning these poles among $\gamma_{i}, \delta_{i}$ and $\epsilon_{i}$. For future reference, we also define the Blaschke product $\mathcal{B}_{\gamma}$ as,

$$
\mathcal{B}_{\gamma}=\prod_{i=1}^{n_{\gamma}} \frac{\left(s-\gamma_{i}\right)}{\left(s+\bar{\gamma}_{i}\right)}
$$

and, $\mathcal{B}_{\delta}$ and $\mathcal{B}_{\epsilon}$ are defined similarly. Here, $G_{s}$ represents the stable version of $G$ defined as $G_{s}=\mathcal{B}_{\gamma} G$.

\section{INTERPOLATION CONSTRAINTS}

In this section, we derive some interpolation constraints, when the system is controlled indirectly. These constraints subsequently limit the achievable performance of the system. We recall that similar constraints for the sensitivity and complementary sensitivity functions were derived by Zames [9].

Proposition 1: For internal stability, $T_{z d}$ must satisfy the following interpolation constraints:

$$
\begin{aligned}
T_{z d}\left(\alpha_{i}\right) & =G_{d}\left(\alpha_{i}\right) \\
T_{z d}\left(\beta_{i}\right) & =G_{d}\left(\beta_{i}\right) \\
T_{z d}\left(\gamma_{i}\right) & =G_{d}\left(\gamma_{i}\right)-G_{s} G_{y, s 1}^{-1} G_{d y}\left(\gamma_{i}\right) \\
T_{z d}\left(\delta_{i}\right) & =G_{d}\left(\delta_{i}\right)-G G_{y, s 2}^{-1} G_{d y, s}\left(\delta_{i}\right) \\
T_{z d}\left(\epsilon_{i}\right) & =G_{d}\left(\epsilon_{i}\right)
\end{aligned}
$$


where $G_{s}=\mathcal{B}_{\gamma} G, G_{y, s 1}=\mathcal{B}_{\gamma} G_{y}, G_{y, s 2}=\mathcal{B}_{\delta} G_{y}$ and $G_{d y, s}=\mathcal{B}_{\delta} G_{d y}$.

Proof: The proofs of (2) and (3) follows from the definition of $T_{z d}$. For (4), first note that $S_{y}\left(\gamma_{i}\right)=0$ and thus $G_{y} K S_{y}\left(\gamma_{i}\right)=1-S_{y}\left(\gamma_{i}\right)=1$. Since $G_{y}=\mathcal{B}_{\gamma}^{-1} G_{y, s 1}$,

$$
\underbrace{\mathcal{B}_{\gamma}^{-1} G_{s}}_{G} K S_{y}\left(\gamma_{i}\right)=G_{s} G_{y, s 1}^{-1}\left(\gamma_{i}\right)
$$

and $T_{z d}\left(\gamma_{i}\right)=G_{d}\left(\gamma_{i}\right)-G_{s} G_{y, s 1}^{-1} G_{d y}\left(\gamma_{i}\right)$. The proof of (5) is similar and thus omitted. For (6), note that $S_{y}\left(\epsilon_{i}\right)=0$ and thus $G K S_{y} G_{d y}\left(\epsilon_{i}\right)=0$, which implies (6).

The interpolation constraints (2)-(6) were earlier derived by Freudenberg et al. [8] using an alternate approach. Each of (2)-(6) implies limitations on the achievable value of $\left\|T_{z d}\right\|_{\infty}$. For example, using maximum modulus principle,

$$
\left\|T_{z d}\right\|_{\infty} \geq \max _{i}\left|G_{d}\left(\alpha_{i}\right)\right|
$$

Thus, whenever $G$ or $G_{d y}$ have RHP zeros, $\left\|T_{z d}\right\|_{\infty}$ cannot be reduced arbitrarily, unless $\left|G_{d}\left(\alpha_{i}\right)\right|=0$, i.e. if $G_{d}$ also contains the RHP-zero. When we have interpolation constraints other than (2), similar lower bounds on $\left\|T_{z d}\right\|_{\infty}$ can be derived. In this case, however, the bound can be loose, as only one constraint is considered at a time. We derive results that consider the effects of the different interpolation constraints together later in this paper.

Note that (4)-(6) can be collectively stated as

$$
T_{z d}(p)=\lim _{s \rightarrow p} \frac{\operatorname{det}(\tilde{G})}{G_{y}}
$$

where is $p$ an unstable pole of $\tilde{G}$ in (1). Then, similar to (7), it follows that whenever $G_{y}$ has RHP poles, $\left\|T_{z d}\right\|_{\infty}>0$, unless $\tilde{G}$ has a transmission zero at the same location as the RHP poles of $G_{y}$. We point out that when $\tilde{G}$ has transmission zeros at the same location as the RHP poles of $G_{y}$, though these constraints may not pose limitations individually, they can be limiting when combined with other constraints.

Remark 1: RHP zeros of $G_{y}$ alone do not pose any fundamental limitation on $T_{z d}$, however, with RHP poles, they can be limiting, as one may expect. This is clear from (4), where $G_{y, s}$ is small and thus $G_{y, s}^{-1}$ is large, when RHP zeros of $G_{y}$ nearly cancel the RHP poles.

It may seem from (6) that the limitations due to the unstable poles of $G_{y}$, which are not shared with $G$ or $G_{d y}$, is the same as RHP zeros of $G$ and $G_{d y}$. This is clearly not true as when $G_{d}\left(\alpha_{i}\right)=0$, a zero controller $K=0$ provides $T_{z d}=0$. On the other hand, whenever the system has unstable poles, $K=0$ is not stabilizing and thus $\left\|T_{z d}\right\|_{i}>0$, $i=2, \infty$. Next, we show that these poles also pose a constraint on the derivative of $T_{z d}$.

Proposition 2: For internal stability, $T_{z d}^{(1)}$ must satisfy the following interpolation constraints:

$$
\begin{aligned}
T_{z d}^{(1)}\left(\epsilon_{i}\right) & =G_{d}^{(1)}\left(\epsilon_{i}\right) \\
& -\left(\frac{1}{2 \operatorname{Re}\left(\epsilon_{i}\right)} \prod_{i=1, i \neq j}^{n_{\epsilon}} \frac{\epsilon_{i}-\epsilon_{j}}{\epsilon_{i}+\bar{\epsilon}_{j}}\right) G G_{y, s}^{-1} G_{d y}\left(\epsilon_{i}\right)
\end{aligned}
$$

where $G_{y, s}=\mathcal{B}_{\epsilon} G$.

Proof: Note that $T_{z d}$ can be alternatively be written as,

$$
T_{z d}=G_{d}-\mathcal{B}_{\epsilon} G G_{y, s}^{-1}\left(I-S_{y}\right) G_{d y}
$$

and thus

$$
\begin{aligned}
T_{z d}^{(1)}=G_{d}^{(1)}-\mathcal{B}_{\epsilon}^{(1)} & G G_{y, s}^{-1}\left(I-S_{y}\right) G_{d y} \\
& -\mathcal{B}_{\epsilon}\left(G G_{y, s}^{-1}\left(I-S_{y}\right) G_{d y}\right)^{(1)}
\end{aligned}
$$

It can be easily verified that

$$
\mathcal{B}_{\epsilon}^{(1)}=\sum_{i=1}^{n_{\epsilon}}\left(\frac{2 \operatorname{Re}\left(\epsilon_{i}\right)}{\left(s+\bar{\epsilon}_{i}\right)^{2}} \prod_{i=1, i \neq j}^{n_{\epsilon}} \frac{s-\epsilon_{j}}{s+\bar{\epsilon}_{j}}\right)
$$

Note that $\left(G G_{y, s}^{-1}\left(I-S_{y}\right) G_{d y}\right)^{(1)}\left(\epsilon_{i}\right)$ is finite. The result follows by substituting for $\mathcal{B}_{\epsilon}^{(1)}$ in (10) and evaluating the resulting expression at $\epsilon_{i}$.

Proposition 1 indicates that the limitations due to RHP poles of $G_{y}$ that are not shared with $G$ or $G_{d y}$ is more severe than those that are shared. For these poles, $\left\|T_{z d}\right\|_{\infty}>0$, even when $\tilde{G}$ has a transmission zero at the same location. We point out that $T_{z d}^{(i)}, i \geq 2$ is dependent on the controller.

To illustrate the limitation due to the derivative constraint (9), we consider that $G_{d}=0, G=G_{d y}=1$, which implies that $T_{z d}=K S_{y}$. Havre and Skogestad [10] have shown that $\left\|K S_{y}\right\|_{\infty} \geq\left|G_{y, s}^{-1}\left(\epsilon_{i}\right)\right|$, where $G_{y, s}=\mathcal{B}_{\epsilon} G_{y}$. The interpolation constraint (6) only requires that $K S_{y}\left(\epsilon_{i}\right)=0$. For simplicity, consider that $G_{y}$ has a single real unstable pole at $\epsilon$. Then, the derivative constraint (9) requires that $\left(K S_{y}\right)^{i}(\epsilon)=(1 / 2 \epsilon) G_{y, s}^{-1}(\epsilon)$. All rational stable functions that satisfy $K S_{y}(\epsilon)=0$ can be represented as

$$
H=\frac{(s-\epsilon)}{(s+\epsilon)} q
$$

where $q$ is a rational stable function. After some simple manipulations, it can be shown the derivative constraint implies that $q(\epsilon)=G_{y, s}^{-1}(\epsilon)$, which requires that $\left\|K S_{y}\right\|_{\infty} \geq$ $\left|G_{y, s}^{-1}(\epsilon)\right|$.

\section{ACHievable $\mathcal{H}_{\infty}$ Performance}

In this section, we use the Nevanlinna-Pick interpolation theory [11] to derive the achievable bound on $\left\|T_{z d}\right\|_{\infty}$. This well-known approach was used extensively for obtaining earlier solutions of the $\mathcal{H}_{\infty}$ control problem; see e.g. [12]. It has earlier been used in [4], [13] for quantification of achievable bounds of sensitivity and complementary sensitivity functions. We begin the discussion by recalling the necessary and sufficient condition that guarantees the existence of rational analytic functions satisfying the interpolation constraints,

$$
\frac{f^{(i)}\left(s_{k}\right)}{i !}=w_{k, i} ; \quad k=1 \cdots n, i=1, \cdots, m_{k}
$$

where $s_{k}$ are distinct points in open RHP and $\operatorname{Re}(f(j \omega))>0$ for all $\omega \in \mathbb{R}$

Lemma $1 \overline{\overline{\bar{F}}}$ ere exists a rational analytic function $f(s)$ with $\|f(s)\|_{\infty} \leq 1$ that satisfies (11), if and only if the corresponding Pick matrix defined as

$$
P=E-W E W^{H}
$$


is positive semi-definite, $P \succeq 0$ [11]. Here

$W=\operatorname{diag}\left(W_{k}\right) ; W_{k}=\left[\begin{array}{cccc}w_{k, 0} & 0 & \cdots & 0 \\ w_{k, 1} & w_{k, 0} & \cdots & 0 \\ \vdots & \ddots & \cdots & 0 \\ w_{k, m_{k}} & \cdots & \cdots & w_{k, 0}\end{array}\right]$

and $E$ is the positive definite solution to the following Lyapunov equation,

$$
A E+E A^{H}=b b^{T}
$$

where

$$
A=\operatorname{diag}\left(A_{k}\right) ; \quad A_{k}=\left[\begin{array}{cccc}
s_{k} & & & \\
1 & s_{k} & & \\
& \ddots & \ddots & \\
& & 1 & s_{k}
\end{array}\right]
$$

where $A_{k}$ is a $m_{k} \times m_{k}$ dimensional matrix and $b=$ $\left[b_{1} \cdots b_{n}\right]^{T}$. Here, $b_{j}$ is an $m_{k}$ dimensional row vector with $b_{j}=\left[\begin{array}{lll}1 & 0 \cdots 0\end{array}\right]$.

To derive the bound on $\left\|T_{z d}\right\|_{\infty}$, as a shorthand notation, we denote

$$
v=\left[\begin{array}{llll}
\alpha & \beta & \gamma & \delta
\end{array}\right]
$$

where $v$ is $n_{v}=\left(n_{\alpha}+n_{\beta}+n_{\gamma}+n_{\delta}\right)$ dimensional vector. Define

$$
Y=\left[\begin{array}{cc}
X & Z \\
Z^{H} & Q
\end{array}\right]
$$

where

$$
\begin{aligned}
& X=\left[X_{i j}\right] ; \quad X_{i j}=\left[\begin{array}{cc}
\frac{1}{\left(\epsilon_{i}+\bar{\epsilon}_{j}\right)} & \frac{-1}{\left(\epsilon_{i}+\bar{\epsilon}_{j}\right)^{2}} \\
\frac{-1}{\left(\epsilon_{i}+\bar{\epsilon}_{j}\right)^{2}} & \frac{2}{\left(\epsilon_{i}+\bar{\epsilon}_{j}\right)^{3}}
\end{array}\right] \\
& Z=\left[Z_{i k}\right] ; \quad Z_{i k}=\left[\begin{array}{c}
\frac{1}{\left(\epsilon_{i}+\bar{v}_{k}\right)} \\
\frac{-1}{\left(\epsilon_{i}+\bar{v}_{k}\right)^{2}}
\end{array}\right] \\
& Q=\left[\frac{1}{v_{k}+\bar{v}_{\ell}}\right]
\end{aligned}
$$

where $X, Z$ and $Q$ are $2 n_{\epsilon} \times 2 n_{\epsilon}, 2 n_{\epsilon} \times n_{v}$ and $n_{v} \times n_{v}$ dimensional matrices, respectively. Further, define

$$
F=\left[\begin{array}{cc}
\operatorname{diag}\left(R_{i}\right) & 0 \\
0 & \operatorname{diag}\left(T_{z d}(v)\right)
\end{array}\right]
$$

where

$$
R_{i}=\left[\begin{array}{cc}
T_{z d}\left(\epsilon_{i}\right) & 0 \\
T_{z d}^{1}\left(\epsilon_{i}\right) & T_{z d}\left(\epsilon_{i}\right)
\end{array}\right] ; \quad i=1, \cdots, n_{\epsilon}
$$

Here, $T_{z d}(v)$ is given by (2)-(5), $T_{z d}(\epsilon)$ by (6) and $T_{z d}^{1}(\epsilon)$ by (9). The next proposition characterizes the achievable value of $\left\|T_{z d}\right\|_{\infty}$

Proposition $3 . \overline{\overline{\bar{\gamma}}}$ ume that the unstable poles of $\tilde{G}$ and the unstable zeros of $G$ and $G_{d y}$ do not lie on the imaginary axis including infinity. If these unstable poles and zeros are non-repeated,

$$
\inf _{K}\left\|T_{z d}\right\|_{\infty}=\bar{\sigma}\left(Y^{-0.5} F Y^{0.5}\right)
$$

where $Y$ and $F$ are defined by (12) and (13), respectively.

Proof: Define $H=(1 / \gamma) \cdot T_{z d}$. It follows that for internal stability, $H$ must satisfy the following interpolation constraints,

$H\left(v_{i}\right)=\frac{1}{\gamma} T_{z d}\left(v_{i}\right) ; H\left(\epsilon_{j}\right)=\frac{1}{\gamma} T_{z d}\left(\epsilon_{j}\right) ; H^{1}\left(\epsilon_{j}\right)=\frac{1}{\gamma} T_{z d}^{1}\left(\epsilon_{j}\right)$

where $i=1, \cdots n_{v}$ and $j=1, \cdots, n_{\epsilon}$. Let

$$
A=\left[\begin{array}{cc}
\operatorname{diag}\left(C_{i}\right) & 0 \\
0 & \operatorname{diag}(v)
\end{array}\right]
$$

where

$$
C_{i}=\left[\begin{array}{cc}
\epsilon_{i} & 0 \\
1 & \epsilon_{i}
\end{array}\right] ; \quad i=1, \cdots, n_{\epsilon}
$$

After some lengthy but simple algebraic manipulations, it can be verified that $Y$ in (12) solves the following Lyapunov equation

$$
A Y+Y A^{H}=b b^{T}
$$

where $b$ is defined similar to Lemma 1 . Based on Lemma 1, there exists analytic $H$ with $\|H\|_{\infty} \leq 1$ that satisfies constraints (15), if and only if

$$
Y-\frac{1}{\gamma^{2}} F Y F^{H} \succeq 0
$$

Since $Y \succ 0, Y$ can be written as, $Y=Y^{0.5}\left(Y^{0.5}\right)^{H}$ and (16) is equivalent to

$$
I-\frac{1}{\gamma^{2}} Y^{-0.5} F Y F^{H}\left(Y^{-0.5}\right)^{H} \succeq 0
$$

which implies

$$
\begin{aligned}
\gamma^{2} & \geq \rho\left(Y^{-0.5} F Y^{0.5}\left(Y^{-0.5} F Y^{0.5}\right)^{H}\right) \\
\gamma & \geq \bar{\sigma}\left(Y^{-0.5} F Y^{0.5}\right)
\end{aligned}
$$

Since $\bar{\sigma}\left(Y^{-0.5} F Y^{0.5}\right)$ is the least achievable value of $\gamma$, the equality follows.

The equality in Proposition 3 implies that there exists (possibly improper) controller such that the bound given by (14) is achieved. Proposition 3 effectively unifies the available achievable bounds on the sensitivity and complementary functions and also $K S$.

Based on Proposition 3, it follows that it is possible to have $\left\|T_{z d}\right\|_{\infty}=0$ (perfect disturbance rejection), if and only if

1) $G$ and $G_{d y}$ have no RHP zeros or $G_{d}$ evaluated at these RHP zeros is zero and

2) $G$ and $G_{d y}$ have no RHP poles or these RHP poles are also transmission zeros of $\tilde{G}$ and

3) $G_{y}$ has no RHP poles by itself (not shared with $G$ or $\left.G_{d y}\right)$.

We notice that the notation leading to Proposition 3 is very messy. The following Corollary provides a lower bound on $\left\|T_{z d}\right\|_{\infty}$, which is simpler to interpret and is more insightful.

Corollary 1: Under the same conditions as Proposition 3,

$$
\inf _{K}\left\|T_{z d}\right\|_{\infty} \geq \bar{\sigma}\left(Q_{1}^{-0.5} F_{1} Q_{1}^{0.5}\right)
$$


where

$$
\begin{aligned}
Q_{1} & =\left[\begin{array}{cc}
Q & 0 \\
0 & Q_{\epsilon}
\end{array}\right] ; \quad Q_{\epsilon}=\left[\frac{1}{\epsilon_{i}+\bar{\epsilon}_{j}}\right] \\
F_{1} & =\left[\begin{array}{cc}
\operatorname{diag}\left(T_{z d}(v)\right) & 0 \\
0 & \operatorname{diag}\left(T_{z d}(\epsilon)\right)
\end{array}\right]
\end{aligned}
$$

Corollary 1 can be shown to be true by ignoring the derivative constraints and following the same procedure as in the proof of Proposition 3. Corollary 1 also follows from (14) directly, as the maximum singular value of a submatrix is always lower than that of the matrix itself. The bound (17) is tight when the the set $\epsilon$ is empty.

Chen [4] provides the achievable bound for the case, when the system is controlled "directly", i.e. $y=z\left(G=G_{y}\right.$ and $\left.G_{d}=G_{d y}\right)$. For direct control, the closed-loop transfer function from $d$ to $z$ is $(I+G K)^{-1} G_{d}=S G_{d}$. Clearly, $S G_{d}\left(\alpha_{i}\right)=G_{d}\left(\alpha_{i}\right)$ and $S G_{d}\left(\gamma_{i}\right)=0$. Based on these interpolation constraints and comparison of (17) with the results in [4], we note that there are no additional limitations on the achievable performance due to the RHP zeros of $G$ (at least for stable systems), however, the RHP poles of $G$ can be very limiting. This justifies the widespread use of indirect control strategy in process industries, which are usually stable. Note that for indirect control, the RHP zeros of $G_{d y}$ can still pose limitations. For indirect control, the most severe additional limitation arises due to the RHP poles of $G_{y}$ that are not shared with $G$. This is expected as additional control effort is required for stabilization. These observations are further illustrated using the following numerical example.

Example 1: To compare the fundamental performance limitations of direct and indirect control, we consider the following simple system,

$G=\frac{(s-1)}{\left(s+p_{1}\right)} \quad G_{y}=\frac{1}{\left(s+p_{1}\right)\left(s+p_{2}\right)} \quad G_{d}=\frac{1}{s+1}$

and $G_{d y}=1$. When, $p_{1}, p_{2}>0, G_{d}(1)=0.5$, which is the achievable value for $\left\|T_{z d}\right\|_{\infty}$ and also $\left\|S G_{d}\right\|_{\infty}$. For the case, when $p_{1}<0$ and $p_{2}>0$, indirect control can be very limiting. For example, for $p_{1}=-2$ and $p_{2}=3$, Corollary 1 implies that the achievable value of $\left\|T_{z d}\right\|_{\infty}$ is 15.348 , where as the corresponding value for direct control is only 1.5. Thus, the unstable pole of $G$ poses 10 fold limitation on the control quality in indirect control as compared to direct control for this example. Further, when $p_{2}=-3$, the limitations become more severe, as the achievable value of $\left\|T_{z d}\right\|_{\infty}$ is 51.95 .

\section{ACHIEVABle $\mathcal{H}_{2}$ PeRformance}

We extend the results of the previous section to cases, where the achievable value of $\left\|T_{z d}\right\|_{2}$ is of interest. Usually, the interpolation constraints are used for analyzing the achievable $\mathcal{H}_{\infty}$ optimal performance; see e.g. [4], [10]. The results in this section show that similar limitations exist on the achievable $\mathcal{H}_{2}$ optimal performance, as one may expect. The next proposition illustrates the effect of RHP poles and zeros on the achievable value of $\left\|T_{z d}\right\|_{2}$.
Proposition 4: Under the same conditions as Proposition 3,

$$
\inf _{K}\left\|T_{z d}\right\|_{2}^{2} \geq\left[T_{z d}(v) \quad T_{z d}(\epsilon)\right] Q_{1}^{-T}\left[T_{z d}(v) \quad T_{z d}(\epsilon)\right]^{H}
$$

where $Q_{1}$ is given by (18).

Proof: To prove (20), we first parameterize the set of rational stable systems that satisfy interpolation constraints (2)(6) followed by the use of model matching theory. For ease of notation, define $t=\left[\begin{array}{ll}v & \epsilon\end{array}\right]$ and

$$
\mathcal{B}_{t}=\prod_{i=1}^{n_{v}+n_{\epsilon}} \frac{\left(s-t_{i}\right)}{\left(s+\bar{t}_{i}\right)} ; \quad \mathcal{B}_{t}^{j}=\prod_{i=1, i \neq j}^{n_{v}+n_{\epsilon}} \frac{\left(s-t_{i}\right)}{\left(s+\bar{t}_{i}\right)}
$$

Then, one possible parametrization of all rational stable functions that satisfy the interpolation constraints (2)-(6) is,

$$
\begin{aligned}
H & =\sum_{i=1}^{n_{v}+n_{\epsilon}} \mathcal{B}_{t}^{i}\left[\mathcal{B}_{t}^{i}\left(t_{i}\right)\right]^{-1} T_{z d}\left(t_{i}\right)+\mathcal{B}_{t} q \\
& =\mathcal{B}_{t}\left(\sum_{i=1}^{n_{v}+n_{\epsilon}} \frac{\left(s+\bar{t}_{i}\right)}{\left(s-t_{i}\right)}\left[\mathcal{B}_{t}^{i}\left(t_{i}\right)\right]^{-1} T_{z d}\left(t_{i}\right)+q\right)
\end{aligned}
$$

where $q$ is a rational stable transfer function and is free to choose. Since $\mathcal{B}_{t}$ is all pass

$$
\|H\|_{2}^{2}=\left\|\sum_{i=1}^{n_{v}+n_{\epsilon}} \frac{\left(s+\bar{t}_{i}\right)}{\left(s-t_{i}\right)}\left[\mathcal{B}_{t}^{i}\left(t_{i}\right)\right]^{-1} T_{z d}\left(t_{i}\right)+q\right\|_{2}^{2}
$$

Defining $L=\sum_{i=1}^{n_{v}+n_{\epsilon}} \frac{2 \operatorname{Re}\left(t_{i}\right)}{\left(s-t_{i}\right)}\left[\mathcal{B}_{t}^{i}\left(t_{i}\right)\right]^{-1} T_{z d}\left(t_{i}\right)$,

$$
\|H\|_{2}^{2}=\left\|L+\sum_{i=1}^{n_{v}+n_{\epsilon}}\left[\mathcal{B}_{i}^{j}\left(t_{i}\right)\right]^{-1} T_{z d}\left(t_{i}\right)+q\right\|_{2}^{2}
$$

Since $L \in \mathcal{R} \mathcal{H}_{2}^{\perp}$, the contribution of the second term in (22) can be negated by appropriate choice of $q$ and

$$
\inf _{q}\|H\|_{2}^{2}=\left\|L^{H}\right\|_{2}^{2}
$$

A state-space realization of $L$ is given as $L \leftrightarrow(A, B, C, D)$, where

$$
\begin{aligned}
& A=\operatorname{diag}\left(t_{i}\right) ; \quad B=\left[2 \operatorname{Re}\left(t_{i}\right)\left[\mathcal{B}_{t}^{i}\left(t_{i}\right)\right]^{-1} T_{z d}\left(t_{i}\right)\right]^{T} \\
& C=1_{n_{v}+n_{\epsilon}} ; \quad D=0
\end{aligned}
$$

where $1_{n_{v}+n_{\epsilon}}$ is an $\left(n_{v}+n_{\epsilon}\right)$ dimensional vector of 1's. The observability gramian for $L^{H}$ with the above realization is given as, $X=\operatorname{diag}\left(B_{i}\right) Q_{1} \operatorname{diag}\left(B_{i}\right)^{H}$ [5] and thus $\left\|L^{H}\right\|_{2}^{2}=$ $B Q_{1} B^{H}$. By expanding the expression for $\left\|L^{H}\right\|_{2}^{2}$,

$$
\begin{aligned}
\left\|L^{H}\right\|_{2}^{2}=T_{z d}(t) \cdot \operatorname{diag}\left(2 \operatorname{Re}\left(t_{i}\right) \mathcal{B}_{t}^{i}\left(t_{i}\right)\right) \cdot Q_{1} \cdot \\
\quad \operatorname{diag}\left(2 \operatorname{Re}\left(t_{i}\right) \mathcal{B}_{t}^{i}\left(t_{i}\right)\right)^{H} \cdot T_{z d}(t)^{H}
\end{aligned}
$$

The result follows by noting that $\operatorname{diag}\left(2 \operatorname{Re}\left(t_{i}\right) \mathcal{B}_{t}^{i}\left(t_{i}\right)\right) \cdot Q_{1}$. $\operatorname{diag}\left(2 \operatorname{Re}\left(t_{i}\right) \mathcal{B}_{t}^{i}\left(t_{i}\right)\right)^{H}=Q_{1}^{-T}$ [5].

In Proposition 4, we have neglected the effect of derivative constraints (9), as this makes the resulting expressions complex and it is difficult to gain any insight. Then (20) provides a lower bound on the achievable value of $\left\|T_{z d}\right\|_{2}$, which is tight for the case where the set $\epsilon$ is empty. Further, 
the lower bound on $\left\|T_{z d}\right\|_{\infty}$ can be alternatively derived using the approach taken in the proof of Proposition 4, i.e. parametrization of all rational stable functions satisfying the interpolation constraints (2)-(6) followed by use of the Nehari extension theorem [12].

The limitations on the achievable value of $\left\|T_{z d}\right\|_{2}$ due to unstable poles and zeros is similar as $\left\|T_{z d}\right\|_{\infty}$. For the system in (19), when $p_{1}, p_{2}>0$ or $G_{y}$ is stable, the achievable performance for the direct and indirect are the same and $\inf _{K}\left\|T_{z d}\right\|_{2}=0.707$. When $p_{1}=-2, p_{2}=3$ such that $G$ shares the unstable pole of $G_{y}$, the achievable $\mathcal{H}_{2}$ optimal performance for direct control is 2.123. On the other hand, $\inf _{K}\left\|T_{z d}\right\|_{2}$ is much larger for indirect control, where based on Proposition 4, we find that inf $K\left\|T_{z d}\right\|_{2}=30.008$. This observation emphasizes that for stable systems, there is no additional fundamental limitation in using indirect control.

\section{FEEDBACK + FEEDFORWARD CONTROL}

We note from Example 1 that the performance of unstable systems under indirect control can be severely limited as compared to direct control. In this section, we show that some of these limitations can be overcome, when the disturbances are measurable. For example, in distillation columns, one of primary disturbance variables is feed flow rate, which can be used to improve the quality of control using feedforward control. We consider that the the disturbance is measurable after being filtered through $M$, which shares its unstable poles with $G_{y}$. Then, the control law is given as,

$$
u=-K_{1} y+K_{2} M d
$$

where $K_{1}$ is the feedback and $K_{2}$ is the feedforward controller. Using (23), the closed-loop transfer function from $d$ to $z$ is

$$
\tilde{T}_{z d}=T_{z d}+G S_{y} K_{2} M
$$

Comparing $\tilde{T}_{z d}$ with $T_{z d}$, we observe that:

1) The interpolation constraint (2) due to the RHP zeros of $G$ also applies to $\tilde{T}_{z d}$. However, $\tilde{T}_{z d}\left(\beta_{i}\right)$ depends on $K_{2}$, unless $M$ also has RHP zeros at $\beta_{i}$.

2) $\tilde{T}_{z d}\left(\gamma_{i}\right)$ and $\tilde{T}_{z d}\left(\delta_{j}\right)$ also depend on $K_{2}$.

3) The RHP poles of $G_{y}$ that are not shared with $G$ and $G_{d y}$ pose interpolation constraints similar to (6), however $\tilde{T}_{z d}^{1}\left(\epsilon_{i}\right)$ depends on $K_{2}$.

In summary, the feedforward controller always overcomes the limitations due to the unstable poles of $G$ and $G_{d y}$ and also partially due to the RHP poles of $G_{y}$ that are not shared with $G$ and $G_{d y}$. In the context of direct control, similar conclusions have been drawn by Havre and Skogestad [10]. In the absence of the derivative constraint, tight bounds on the achievable value of $\left\|\tilde{T}_{z d}\right\|_{i}$ can be derived, similar to Corollary 1 for $i=\infty$ and Proposition 4 for $i=2$.

Example 2: We revisit Example 1, where the unstable poles of $G$ and $G_{y}$ posed severe limitations on the achievable value of $\left\|\tilde{T}_{z d}\right\|_{\infty}$, as compared to direct control, with $(M=$ $1)$. When the disturbance can be measured, the poles of $G$ do not pose any limitations. Thus $\inf _{K}\left\|\tilde{T}_{z d}\right\|_{\infty}=0.5$ for $p_{1}=-2, p_{2}=3$, which is the same as achievable for the stable system $\left(p_{1}, p_{2}>0\right)$ without feedforward control. Similarly, when $p_{2}=-3$ the lack of the derivative constraint for feedback + feedforward control, drastically reduces the achievable value of $\left\|\tilde{T}_{z d}\right\|_{\infty}$ from 51.95 to 0.683 . For comparison, we determine that for direct feedback + feedforward control, the achievable value of $\mathcal{H}_{\infty}$ optimal control is 0.5 for all cases.

\section{CONCLUSIONS}

We studied the achievable performance for systems under indirect control, where all the signals in the system are scalar. It is shown that in addition to the regular interpolation constraints, the derivative of closed-loop transfer function is also fixed at the unstable poles not observable in the primary controlled variable. We proposed bounds on the achievable performance, which is tight in the $\mathcal{H}_{\infty}$ control framework, and also in the $\mathcal{H}_{2}$ control framework, except when some of the unstable poles are observable only in the transfer function from the input to the secondary controlled variable.

A surprising result is that for stable systems, RHP-zeros of $G$ do not pose any additional limitations on the achievable performance, as compared to direct control. For unstable systems, however, systems under indirect control can have severe limitations. When the disturbance is measurable, an additional feedforward controller can overcome the limiting effect of unstable poles observable in the primary controlled variable.

\section{REFERENCES}

[1] S. Skogestad and I. Postlethwaite, Multivariable Feedback Control: Analysis and Design, 1st ed. Chichester, UK: John Wiley \& Sons, 1996.

[2] A. Faanes and S. Skogestad, "pH-neutralization: Integrated process and control design," Computers Chem. Engng., vol. 28, no. 8, pp. 1475-1487, 2004.

[3] G. Stein, "Respect the unstable," IEEE Control Systems Magazine, vol. 23, no. 4, pp. 12-25, 2003.

[4] J. Chen, "Logarithmic integrals, interpolation bounds and performance limitations in MIMO feedback systems," IEEE Trans. Automat. Contr., vol. 45, no. 6, pp. 1098-1115, 2000.

[5] V. Kariwala, S. Skogestad, J. F. Forbes, and E. S. Meadows, "Achievable input performance of linear systems under feedback control," 2005, To be published in Int. J. Control.

[6] M. M. Seron, J. H. Braslavsky, and G. C. Goodwin, Fundamental Limitations in Filtering and Control, ser. Communications and Control Engineering. London: Springer-Verlag, 1997.

[7] J. Chen and R. H. Middleton, "New development and applications in performance limitation of feedback control," IEEE Trans. Automat. Contr., vol. 48, no. 8, p. 1297, 2003.

[8] J. S. Freudenberg, C. V. Hollot, R. H. Middleton, and V. Toochinda, "Fundamental design limitations of the general control configuration," IEEE Trans. Automat. Contr., vol. 48, no. 8, pp. 1355-1370, 2003.

[9] G. Zames, "Feedback and optimal sensitivity: Model reference transformations, multiplicative seminorms, and approximate inverses," IEEE Trans. Automat. Contr., vol. 26, no. 2, pp. 301-320, 1981.

[10] K. Havre and S. Skogestad, "Achievable performance of multivariable systems with unstable zeros and poles," Int. J. Control, vol. 74, no. 11, pp. 1131-1139, 2001.

[11] J. A. Ball, I. Gohberg, and L. Rodman, Interpolation of Rational Matrix Functions, ser. Operator Theory: Advances and Applications. Basel, Germany: Birkhäuser Verlag, 1990, vol. 45.

[12] B. A. Francis, A Course in $\mathcal{H}_{\infty}$ Control Theory, ser. Lecture notes in Control and Information Science. Berlin: Springer-Verlag, 1987, vol. 88.

[13] S. D. O'Young and B. A. Francis, "Sensitivity tradeoffs for multivariable plants," IEEE Trans. Automat. Contr., vol. 30, no. 7, pp. 625-632, 1985. 